\title{
Semilocalized Approach to Investigation of Chemical Reactivity
}

\author{
V. GINEITYTE \\ Institute of Theoretical Physics and Astronomy, Vilnius University, \\ Gostauto 12, 2600 Vilnius, Lithuania
}

Received 5 November 2002; accepted 8 May 2003

DOI 10.1002/qua.10704

\begin{abstract}
Application of the power series for the one-electron density matrix (Gineityte, V., J Mol Struct Theochem 1995, 343, 183) to the case of two interacting molecules is shown to yield a semilocalized approach to investigate chemical reactivity, which is characterized by the following distinctive features: (1) Electron density (ED) redistributions embracing orbitals of the reaction centers of both molecules and of their neighboring fragments are studied instead of the total intermolecular interaction energy; (2) the ED redistributions are expressed directly in the basis of fragmental orbitals (FOs) without passing to the basis of delocalized molecular orbitals (MOs) of initial molecules; (3) terms describing the ED redistributions due to an intermolecular contact arise as additive corrections to the purely monomolecular terms and thereby may be analyzed independently; (4) local ED redistributions only between orbitals of the reaction centers of both molecules are described by lower-order terms of the power series, whereas those embracing both the reaction centers and their neighborhoods are represented by higherorder terms. As opposed to the standard perturbative methods based on invoking the delocalized (canonical) MOs of isolated molecules, the results of the approach suggested are in-line with the well-known intuition-based concepts of the classic chemistry concerning reactivity, namely, with the assumption about different roles of the reaction center and of its neighborhood in a chemical process, with the expectation about extinction of the indirect influence of a certain fragment (substituent) when its distance from the reaction center grows, etc. Such a parallelism yields quantum chemical analogs for the classic concepts and thereby gives an additional insight into their nature. The scope of validity of these concepts also is discussed. Applicability of the approach suggested to specific chemical problems is illustrated by a brief consideration of the $\mathrm{S}_{N} 2$ and $\mathrm{Ad}_{E} 2$ reactions. (c) 2003 Wiley Periodicals, Inc. Int J Quantum Chem 94: 302-316, 2003
\end{abstract}

Key words: chemical reactivity; reaction center; intermolecular interaction; electron density distribution; one-electron density matrix 


\section{Introduction}

$\mathbf{0}$ rganic molecules are usually considered as consisting of separate fragments (functional groups) that undergo characteristic reactions. This fundamental principle of the classic chemistry forms the basis of classification of both chemical compounds and their reactions [1-6]. The main point here is that certain types of reactions are expected to be a distinctive feature of the whole class of molecules containing the same functional group(s).

An alternative form of this principle consists of ascribing different roles for separate fragments of a certain compound in the given reaction. Thus, a definite functional group is regarded as taking part in the given process directly and it is usually referred to as the reaction center [1]. The direct participation implies that some bonds are broken and new bonds are formed just within this fragment. Moreover, a local spatial contact only between the relevant reaction centers of two molecules is expected to be sufficient for the given reaction to begin. So far as the remaining parts of molecules are concerned, these are supposed to participate in the process indirectly by exerting certain electron-donating or -accepting effects upon the respective reaction centers, and the extents of these effects are usually considered to be different at various stages of the reaction $[2,6,7]$. As a result, the relative rate of the process is expected to be altered but not the mechanism of the reaction. Extinction of the indirect influence when the distance between the given fragment and the reaction center grows also is among the expectations. The above-described local point of view to chemical reactivity forms the basis of the well-known powerful LFER method based on the linear free energy relationship [3, 4, 6].

Among quantum chemical approaches to reactivity, however, the concept of a nonlocal interaction between entire molecules proves prevalent. This equally refers both to the pioneering contributions based on the simple Hückel theory [8-18] and to the perturbative approaches of a considerably higher level of sophistication developed later [1928] and involving exact many-electron Hamiltonian operators. Mathematically, the above-mentioned point of view is represented by passing to the basis of delocalized (canonical) molecular orbitals (CMOs) either of initial molecules or of a supposed transition state [1, 29-32]. The popular qualitative
HOMO/LUMO concept $[22,30,33,34]$ also reflects the same delocalized point of view.

Despite their popularity, however, the delocalized approaches could not entirely supplant the intuition-based idea about the direct and indirect participation of separate fragments in chemical processes, especially in those between nonconjugated molecules. For example, simple local models are used for interpretation of $S_{N} 2$ processes between nucleophiles and substituted alkanes [1, 6, 29], viz. a local interaction is considered between the electron-donating orbital of nucleophile and the antibonding orbital of the nucleofuge-containing bond. The influence of the remaining bonds of both participants of the reaction is entirely ignored in these models.

In this article, we are about to suggest a semilocalized perturbative approach to investigate chemical reactivity, which is intended for filling the gap between the standard delocalized approaches and their oversimplified strictly localized alternatives. At the same time, we seek to obtain the quantum chemical analogs for the above-discussed classic concepts (especially for the direct and indirect participation of certain fragment in a chemical process) and thereby give an additional insight into the nature of these concepts and establish the scope of their validity.

In this connection, an intermolecular interaction is studied directly in terms of orbitals localized on separate fragments of participating molecules (fragmental orbitals) without passing to the basis of CMOs. As opposed to the simple local models, however, orbitals of all fragments of reacting systems are included in the basis set.

Passing to the basis of CMOs of isolated molecules underlying the standard approaches implies an initial diagonalization of respective Fockian (or Hückel-type Hamiltonian) matrices and thereby an exact taking into account of the intramolecular interactions. The intermolecular interactions, however, are treated perturbatively in the second step of the theory. By contrast, both intra- and intermolecular interactions between fragmental orbitals (FOs) are considered perturbatively in our approach.

The standard approaches are usually based on investigation of total energies of two interacting molecules. This characteristic, however, is hardly the most suitable one for revealing local consequences of an intermolecular contact. In this connection, electron density redistributions among FOs due to an intermolecular contact are studied in this 
article instead of alterations in the total energies. A simple interrelation between these two characteristics established recently [35] may be invoked when comparing our approach to those based on total energies.

When analyzing the most important peculiarities of the above-specified electron density redistributions, it is advisable to use some algebraic expressions for the relevant one-electron density matrix (DM). For a Hückel-type model Hamiltonian matrix represented in the basis of FOs, the expressions for the DM of a general nature have been derived by means of a direct solution of the so-called commutation equation in the form of power series without invoking CMOs [36-43]. Moreover, no specifying either of the number of FOs or of their constitution was required there. As a result, numerous common features of charge redistributions were found for particular types of molecules [38, 41, 43]. As an initial step of the theory, we confine ourselves here to application of these expressions to the case of two interacting molecules. General results concerning chemical reactivity also are anticipated to follow from this study.

The only condition imposed in Refs. [36-43] on the initial basis set was the possibility of revealing two subsets of basis functions containing the initially occupied and initially vacant FOs, respectively, so that the energy intervals between orbitals of different subsets exceed the intersubset interactions considerably. For saturated organic molecules, it is the basis of localized two-center bond orbitals along with lone pair orbitals that was shown to satisfy this requirement $[37,38]$. In the case of the $\pi$-electron subsystems of aliphatic conjugated compounds, two-center bond orbitals of the initially double bonds meet the same condition fairly well [40]. Finally, if the system under study contains aromatic (or heteroaromatic) fragments (e.g., the phenyl ring in substituted benzenes) [41, 43] the CMOs of respective aromatic hydrocarbons (of benzene, respectively) may be used to represent them.

The above-enumerated groups of atoms (the single and double bonds, phenyl rings, etc.) coincide with the smallest quasitransferable fragments of organic molecules, which will be further referred to as elementary fragments. The respective orbitals (FOs) will be in general defined as eigenfunctions of separate Hamiltonian matrix blocks corresponding to subsets of atomic orbitals (AOs) localized on these fragments.
Note that members of the power series for the DM $P$ to within the fourth order inclusive prove to be essential when describing electron density redistribution among localized basis orbitals [40, 43-45] in contrast to the case of delocalized orbitals (CMOs) of initial molecules, where confinement to terms of second order is sufficient $[29,46]$. In this connection, a brief summary of corrections $P_{(k)}$ to within $k=4$ is given in Section 2 along with discussing a convenient way of representing charge redistribution in terms of partial transferred populations between FOs of opposite initial occupation. Thereupon, we apply the general expressions for $P_{(k)}$ to the case of two interacting molecules and prove an important additivity relation for the total DM with respect to intra- and intermolecular contributions (Section 3). This relation forms the basis of further derivation of expressions for alterations in the partial transferred populations between FOs due to an intermolecular contact (Section 4). Analysis of these expressions also is carried out in the same section. Specific examples of applicability of the approach suggested are discussed briefly in Section 5.

\section{General Expressions for the One-Electron DM: Two-Orbital Nature of Charge Redistribution}

Let the total basis set of FOs $\{\varphi\}$ to be divided into two subsets $\left\{\varphi_{(+)}\right\}$and $\left\{\varphi_{(-)}\right\}$containing the initially occupied and initially vacant orbitals, respectively [36], further abbreviated as IOFOs and IVFOs. The intersubset resonance parameters are assumed to be first-order terms vs. the intersubset energy intervals. The actual number of molecules is not essential here.

Then, the Hückel-type one-electron Hamiltonian matrix $H$ of our molecular system may be expressed as a sum of first- and second-order matrices, i.e.,

$$
H=H_{(0)}+H_{(1)}
$$

where

$$
H_{(0)}=\left|\begin{array}{cc}
E_{(+)} & 0 \\
0 & -E_{(-)}
\end{array}\right|, \quad H_{(1)}=\left|\begin{array}{cc}
S & R \\
R^{+} & Q
\end{array}\right| .
$$

The diagonal blocks (submatrices) $E_{(+)}+S$ and $-E_{(-)}+Q$ of the matrix $H$ contain intrasubset interactions (resonance parameters) along with 
one-electron energies of FOs, while the off-diagonal block $R$ involves intersubset interactions. The minus sign in front of $E_{(-)}$is introduced for convenience, and the superscript + designates the transposed matrix. It is also seen that zero-order intrasubset interactions are in general allowed in Eq. (2) and these are included into submatrices $E_{(+)}$ and $E_{(-)}$.

The one-electron DM $P$ corresponding to the Hamiltonian matrix $H$ of Eqs. (1) and (2) has been obtained in Refs. [36-43] as a sum over $k$ of corrections $P_{(k)}$ of the following constitution:

$$
P_{(k)}=-2\left|\begin{array}{cc}
X_{(k)+} & G_{(k)} \\
G_{(k)}^{+} & -X_{(k)-}
\end{array}\right|,
$$

where $k=0,1,2 \ldots$ stands for the order parameter. The submatrices $-2 X_{(k)+}$ and $2 X_{(k)-}$ of the matrix $P_{(k)}$ contain the $k$ th-order corrections to occupation numbers of basis orbitals and intrasubset bond orders, while the off-diagonal blocks $-2 G_{(k)}$ involve the respective corrections to intersubset bond orders. Moreover, the blocks $X_{(k)+}$ and $X_{(k)-}$ have been expressed in Refs. [36-43] in terms of matrices $G_{(k-1)}, G_{(k-2)}$, etc., taking the off-diagonal positions in the previous corrections $P_{(k-1)}, P_{(k-2)}$, etc. Thus, the first four blocks $X_{(k)+}$ and $X_{(k)-}$ take the form

$$
\begin{aligned}
X_{(0)+}=-I, X_{(0)-}=0, X_{(1)+}=X_{(1)-}=0, \\
X_{(2)+}=G_{(1)} G_{(1)}^{+}, X_{(2)-}=G_{(1)}^{+} G_{(1),} \\
X_{(3)+}=G_{(1)} G_{(2)}^{+}+G_{(2)} G_{(1)}^{+}, X_{(3)-}=G_{(1)}^{+} G_{(2)}+G_{(2)}^{+} G_{(1)}, \\
X_{(4)+}=G_{(1)} G_{(3)}^{+}+G_{(3)} G_{(1)}^{+}+G_{(2)} G_{(2)}^{+}+G_{(1)} G_{(1)}^{+} G_{(1)} G_{(1)}^{+}, \\
X_{(4)-}=G_{(1)}^{+} G_{(3)}+G_{(3)}^{+} G_{(1)}+G_{(2)}^{+} G_{(2)} \\
\quad+G_{(1)}^{+} G_{(1)} G_{(1)}^{+} G_{(1)},
\end{aligned}
$$

where $I$ is the unit matrix. The off-diagonal blocks $G_{(k)}$, in turn, meet the matrix equations of the form

$$
E_{(+)} G_{(k)}+G_{(k)} E_{(-)}+W_{(k)}=0,
$$

where

$$
\begin{aligned}
W_{(1)}=R, W_{(2)}=S G_{(1)} & -G_{(1)} Q, \\
W_{(3)}=S G_{(2)}-G_{(2)} Q & -\left(R G_{(1)}^{+} G_{(1)}+G_{(1)} G_{(1)}^{+} R\right), \\
W_{(4)}=S G_{(3)}-G_{(3)} Q & -\left[R\left(G_{(1)}^{+} G_{(2)}+G_{(2)}^{+} G_{(1)}\right)\right. \\
& \left.+\left(G_{(1)} G_{(2)}^{+}+G_{(2)} G_{(1)}^{+}\right) R\right] .
\end{aligned}
$$

The solution of Eq. (6) takes the form of an integral [36, 39, 42, 47]:

$$
G_{(k)}=\int_{0}^{\infty} \exp \left(E_{(+)} t\right) W_{(k)} \exp \left(E_{(-)} t\right) d t \equiv\left(E_{(+)}\left|W_{(k)}\right| E_{(-)}\right),
$$

where the last expression represents a simplified notation of the same integral.

Given that the zero-order blocks $E_{(+)}$and $E_{(-)}$are diagonal matrices, algebraic expressions for particular elements $G_{(k) i l}$ may be obtained [36, 37], viz.

$$
G_{(k) i l}=-\frac{W_{(k) i l}}{E_{(+) i}+E_{(-) l}},
$$

where $E_{(+) i} \equiv E_{(+) i i}$ and $E_{(-) l} \equiv E_{(-) l l}$. The first-order element $G_{(1) i l}$ follows from Eq. (8) straightforwardly after replacing $W_{(1) i l}$ by the relevant resonance parameter $R_{i l}$. This element describes the direct (through-space) interaction between the IOFO $\varphi_{(+) i}$ and the IVFO $\varphi_{(-) l}$. Accordingly, the second-order element $G_{(2) i l}$ takes the form

$$
\begin{aligned}
G_{(2) i l}= & \frac{1}{E_{(+) i}+E_{(-) l}} \\
& \times\left(\sum_{j}^{\text {IOFOs }} \frac{S_{i j} R_{j l}}{E_{(+) j}+E_{(-) l}}-\sum_{r}^{\text {IVFOs }} \frac{R_{i r} Q_{r l}}{E_{(+) i}+E_{(-) r}}\right)
\end{aligned}
$$

and represents the indirect interaction of the same orbitals by means of a single mediator (either an IOFO $\varphi_{(+) j}$ or an IVFO $\left.\varphi_{(-) r}\right)$. Nonzero values of resonance parameters between the mediator and the interacting orbitals are required to ensure a significant value of the indirect interaction $G_{(2) i l}$.

Similarly, the third-order element $G_{(3) i l}$ describes an analogous indirect interaction by means of two mediators. Because of the cumbersome form of the relevant expression [43], it is not given here. Instead, we confine ourselves to the notation that a pair of basis orbitals of any initial occupation (e.g., certain FOs $\varphi_{x}$ and $\varphi_{y}$ ) is able to contribute to the total matrix element $G_{(3) i l}$ considerably if resonance parameters between pairs of orbitals $\left(\varphi_{(+) i}, \varphi_{x}\right),\left(\varphi_{x}, \varphi_{y}\right)$, and $\left(\varphi_{y}, \varphi_{(-) l}\right)$ take significant values.

If $E_{(+)}$and $E_{(-)}$are arbitrary negative definite matrices [42], no local relations like that of Eq. (8) 
may be established between elements $G_{(k) i l}$ and $W_{(k) i l}$. Hence, any element $G_{(k) i l}$ (including $G_{(1) i l}$ ) describes a nonlocal interaction in this case. Nevertheless, particular basis functions (viz. $\varphi_{(+) i}$ and $\left.\varphi_{(-) l}\right)$ correspond to the subscripts $i$ and $l$ of an element $G_{(k) i l}$.

The populations of an IOFO $\varphi_{(+) i}$ and of an IVFO $\varphi_{(-) p}$ may be evidently defined as sums over $k$ of respective diagonal elements of matrices $-2 X_{(k)+}$ and $2 X_{(k)-}$. The latter, in turn, consist of sums of products, each of them containing two matrices $G_{(k)}$ [see Eq. (4)]. This implies that the elements $X_{(k)+, i i}$ and $X_{(k)-, p p}$ and thereby the above-mentioned populations $\left(x_{(+) i}\right.$ and $\left.x_{(-) p}\right)$ may be expressed as sums of increments of individual orbitals of the opposite subset. After summing up these expressions over $k$, we then obtain

$$
x_{(+) i}=2-\sum_{p}^{\mathrm{IVFO}} x_{(+) i,(-) p}, x_{(-) m}=\sum_{i}^{\text {IOFOs }} x_{(-) p,(+) i r}
$$

where 2 represents the initial occupation number of the IOFO $\varphi_{(+) i}$ and

$$
\begin{aligned}
x_{(+) i,(-) p}= & x_{(-) p,(+) i} \\
= & 2\left[\left(G_{(1) i p}\right)^{2}+2 G_{(1) i p} G_{(2) i p}+2 G_{(1) i p} G_{(3) i p}\right. \\
& \left.+\left(G_{(2) i p}\right)^{2}+G_{(1) i p}\left(G_{(1)} G_{(1)}^{+} G_{(1)}\right)_{i p}+\ldots\right] .
\end{aligned}
$$

The expression for $x_{(+) i}$ of Eq. (10) indicates that the increment $x_{(+) i,(-) p}$ describes the partial population donated by the IOFO $\varphi_{(+) i}$ to the IVFO $\varphi_{(-) p}$. Similarly, $x_{(-) p,(+) i}$ represents the partial population acquired by the IVFO $\varphi_{(-) p}$ from the IOFO $\varphi_{(+) i}$ whereas the first relation of Eq. (11) is nothing more than the charge conservation condition.

Therefore, the charge redistribution between FOs due to their interaction may be represented in terms of partial contributions, each of them referring to a pair of FOs of opposite initial occupation, i.e., an IOFO and an IVFO. It should be emphasized, however, that any partial increment $x_{(+) i,(-) p}$ defined by Eq. (11) in general is a nonlocal characteristic. Indeed, it contains indirect interactions of orbitals $\varphi_{(+) i}$ and $\varphi_{(-) p}$ and thereby depends implicitly of the whole set of FOs.

\section{Additivity of Intra- and Intermolecular Contributions to the One-Electron DM of Two Interacting Molecules}

The above-described form of the DM may be easily applied to the case of two interacting molecules $A$ and $B$. To this end, the subset of IOFOs $\left\{\varphi_{(+)}\right\}$will be further subdivided into two parts $\left\{\varphi_{(+)}^{(a)}\right\}$ and $\left\{\varphi_{(+)}^{(b)}\right\}$ referring to molecules $A$ and $B$, respectively. Similarly, the subset of IVFOs will consist of two parts $\left\{\varphi_{(-)}^{(a)}\right\}$ and $\left\{\varphi_{(-)}^{(b)}\right\}$. As a result, the total basis set may be represented in terms of four subsets, i.e., $\{\varphi\}=\left\{\left\{\varphi_{(+)}^{(a)}\right\},\left\{\varphi_{(+)}^{(b)}\right\},\left\{\varphi_{(-)}^{(a)}\right\},\left\{\varphi_{(-)}^{(b)}\right\}\right\}$.

The blocks (submatrices) of matrices $H_{(0)}$ and $H_{(1)}$ will be subdivided accordingly. Thus, the zeroorder blocks $E_{(+)}$and $E_{(-)}$take the form

$$
\begin{aligned}
& E_{(+)}=\left|\begin{array}{cc}
E_{(+)}^{(a)} & 0 \\
0 & E_{(+)}^{(b)}
\end{array}\right| \equiv E_{(+)}^{(a)} \oplus E_{(+)}^{(b)} \\
& E_{(-)}=\left|\begin{array}{cc}
E_{(-)}^{(a)} & 0 \\
0 & E_{(-)}^{(b)}
\end{array}\right| \equiv E_{(-)}^{(a)} \oplus E_{(-)}^{(b)}
\end{aligned}
$$

where the symbol $\oplus$ designates the direct sum of matrices. For the first-order submatrices $S, R$, and $Q$ we obtain

$$
\begin{aligned}
& S=S_{a} \oplus S_{b}+\delta S, \\
& R=R_{a} \oplus R_{b}+\delta R, \\
& Q=Q_{a} \oplus Q_{b}+\delta Q .
\end{aligned}
$$

The blocks of Eqs. (12) and (13) denoted by suband superscripts $(a)$ and $(b)$ contain interactions (resonance parameters) inside the molecules $A$ and $B$, respectively. The remaining parts of matrices $S$, $R$, and $Q$ (supplemented by the symbol $\delta$ ) consist of intermolecular interactions and take an antiblockdiagonal form as follows:

$$
\delta S=\left|\begin{array}{cc}
0 & M \\
M^{+} & 0
\end{array}\right|, \delta R=\left|\begin{array}{cc}
0 & K \\
L & 0
\end{array}\right|, \delta Q=\left|\begin{array}{cc}
0 & T \\
T^{+} & 0
\end{array}\right| .
$$

Equations (12)-(14) also involve an additional assumption that intermolecular resonance parameters are first-order terms comparable to those contained within the matrices $R_{a}$ and $R_{b}$.

Let us prove first the following property of matrices $G_{(k)}$ : 


$$
G_{(k)}=G_{(k)}^{(a)} \oplus G_{(k)}^{(b)}+\delta G_{(k)}
$$

where $G_{(k)}^{(a)}$ and $G_{(k)}^{(b)}$ are purely monomolecular terms and $\delta G_{(k)}$ is a correction describing the increment of the intermolecular interaction. The latter vanishes if the intermolecular resonance parameters contained within matrices $\delta S, \delta R$, and $\delta Q$ turn to zero.

Let us start with first-order terms $(k=1)$. The block-diagonal structure of matrices $E_{(+)}$and $E_{(-)}$ seen from Eq. (12) implies that matrices $\exp \left(E_{(+)} t\right)$ and $\exp \left(E_{(-)} t\right)$ are block-diagonal too, e.g.,

$$
\exp \left(E_{(+)} t\right)=\exp \left(E_{(+)}^{(a)} t\right) \oplus \exp \left(E_{(+)}^{(b)} t\right) .
$$

Then, from Eqs. (6) and (7) it follows that the matrix $G_{(1)}$ complies with the form shown in Eq. (15), where

$$
G_{(1)}^{(a)}=\left(E_{(+)}^{(a)}\left|R_{a}\right| E_{(-)}^{(a)}\right), G_{(1)}^{(b)}=\left(E_{(+)}^{(b)}\left|R_{b}\right| E_{(-)}^{(b)}\right) .
$$

Moreover, the intermolecular increment $\delta G_{(1)}$ also is of the antiblock-diagonal constitution like that of Eq. (14) and contains matrices $G_{(1)}^{(K)}$ and $G_{(1)}^{(L)}$ defined as follows:

$$
G_{(1)}^{(K)}=\left(E_{(+)}^{(a)}|K| E_{(-)}^{(b)}\right), G_{(1)}^{(L)}=\left(E_{(+)}^{(b)}|L| E_{(-)}^{(a)}\right)
$$

in its off-diagonal positions. It is seen that blocks of Eq. (18) turn into zero matrices if the intermolecular resonance parameters contained within matrices $K$ and $L$ vanish. Thus, the above-formulated property is proven for the first-order matrix $G_{(1)}$.

Let us turn now to the second-order matrix $G_{(2)}$. To this end, the above-derived form of the matrix $G_{(1)}$ should be used when constructing the matrix $W_{(2)}$ in accordance with Eq. (6). As a result, an increment of the form $W_{(2)}^{(a)} \oplus W_{(2)}^{(b)}$ will be obtained from the intramolecular terms $S_{a} \oplus S_{b}, Q_{a} \oplus Q_{b}$, and $G_{(1)}^{(a)} \oplus G_{(1)}^{(b)}$. On the other hand, contributions containing both one and two $\delta$ symbols yield an intermolecular increment $\delta W_{(2)}$, which turns into a zero matrix if $M=T=K=L=0$. Hence, the matrix $G_{(2)}$ also complies with the form of Eq. (15) if Eq. (16) along with its counterpart for $\exp \left(E_{(-)} t\right)$ are in addition taken into consideration.

As opposed to the first-order intermolecular term $\delta G_{(1)}$, however, the second-order term $\delta G_{(2)}$ contains four nonzero submatrices. Let the subscripts $a a, b b, a b$, and $b a$ be ascribed to individual blocks of the matrix $\delta G_{(2)}$ as follows:

$$
\delta G_{(2)}=\left|\begin{array}{ll}
\delta G_{(2) a a} & \delta G_{(2) a b} \\
\delta G_{(2) b a} & \delta G_{(2) b b}
\end{array}\right| .
$$

We then obtain

$$
\begin{aligned}
\delta G_{(2) a a}= & \left(E_{(+)}^{(a)}\left|\left(M G_{(1)}^{(L)}-G_{(1)}^{(K)} T^{+}\right)\right| E_{(-)}^{(a)}\right), \\
\delta G_{(2) b b}= & \left(E_{(+)}^{(b)}\left|\left(M^{+} G_{(1)}^{(K)}-G_{(1)}^{(L)} T\right)\right| E_{(-)}^{(b)}\right), \\
\delta G_{(2) a b}= & \left(E_{(+)}^{(a)} \mid\left[\left(S_{a} G_{(1)}^{(K)}-G_{(1)}^{(K)} Q_{b}\right)\right.\right. \\
& \left.\left.+\left(M G_{(1)}^{(b)}-G_{(1)}^{(a)} T\right)\right] \mid E_{(-)}^{(b)}\right), \\
\delta G_{(2) b a}= & \left(E_{(+)}^{(b)} \mid\left[\left(S_{b} G_{(1)}^{(L)}-G_{(1)}^{(L)} Q_{a}\right)\right.\right. \\
& \left.\left.+\left(M^{+} G_{(1)}^{(a)}-G_{(1)}^{(b)} T^{+}\right)\right] \mid E_{(-)}^{(a)}\right),
\end{aligned}
$$

where notations like those of Eq. (7) are used for integrals.

The third-order correction $G_{(3)}$ also may be treated similarly, and the relation of Eq. (15) for $k=$ 3 may be verified without difficulties. Moreover, submatrices of the relevant intermolecular part $\left(\delta G_{(3)}\right)$ may be denoted by subscripts as shown in Eq. (19) and examplified by the following two expressions:

$$
\begin{aligned}
\delta G_{(3) a a}= & \left(E_{(+)}^{(a)} \mid\left[\left(S_{a} \delta G_{(2) a a}-\delta G_{(2) a a} Q_{a}\right)\right.\right. \\
& +\left(M \delta G_{(2) b a}-\delta G_{(2) a b} T^{+}\right)-\left(K G_{(1)}^{(K)+} G_{(1)}^{(a)}\right. \\
& +K G_{(1)}^{(b)+} G_{(1)}^{(L)}+G_{(1)}^{(a)} G_{(1)}^{(L)+} L+G_{(1)}^{(K)} G_{(1)}^{(b)+} L \\
+ & \left.\left.\left.R_{a} G_{(1)}^{(L)+} G_{(1)}^{(L)}+G_{(1)}^{(K)} G_{(1)}^{(K)+} R_{a}\right)\right] \mid E_{(-)}^{(a)}\right), \quad(21) \\
\delta G_{(3) a b}= & \left(E_{(+)}^{(a)} \mid\left[\left(S_{a} \delta G_{(2) a b}-\delta G_{(2) a b} Q_{b}\right)\right.\right. \\
& +\left(M \delta G_{(2) b b}-\delta G_{(2) a a} T\right)-\left(R_{a} G_{(1)}^{(a)+} G_{(1)}^{(K)}\right. \\
& +R_{a} G_{(1)}^{(L)+} G_{(1)}^{(b)}+G_{(1)}^{(a)} G_{(1)}^{(L)+} R_{b} \\
& +G_{(1)}^{(K)} G_{(1)}^{(b)+} R_{b}+K G_{(1)}^{(K)+} G_{(1)}^{(K)} \\
& \left.\left.\left.+G_{(1)}^{(K)} G_{(1)}^{(K)+} K\right)\right] \mid E_{(-)}^{(b)}\right) .
\end{aligned}
$$

Given that $E_{(+)}$and $E_{(-)}$are diagonal matrices, elements of corrections $\delta G_{(2)}$ and $\delta G_{(3)}$ may be interpreted as indirect interactions of basis orbitals as discussed in Section 2. Moreover, the emergence of nonzero intramolecular blocks $\delta G_{(2) a a}, \delta G_{(2) b b}$, $\delta G_{(3) a a}$ and $\delta G_{(3) b b}$ may be accounted for in this case by new indirect interactions arising between orbitals of the same molecule owing to additional mediators offered by another molecule. For example, 
the element $\delta G_{(2) a a, i p}$ defined by Eq. (20) takes the form

$$
\begin{aligned}
& \delta G_{(2) a a, i p}=\frac{1}{E_{(+) i}^{(a)}+E_{(-) p}^{(a)}} \\
& \quad \times\left(\sum_{j}^{\operatorname{IOFOs}(B)} \frac{M_{i j} L_{j p}}{E_{(+) j}^{(b)}+E_{(-) p}^{(a)}}-\sum_{q}^{\operatorname{IVFOS}(B)} \frac{K_{i q} T_{q p}}{E_{(+) i}^{(a)}+E_{(-) q}^{(b)}}\right)
\end{aligned}
$$

and describes the indirect interaction between the IOFO $\varphi_{(+) i}^{a}$ and the IVFO $\varphi_{(-) p}^{a}$ of molecule $A$ by means of a single mediator among orbitals of molecule $B$.

The specific structure of the principal matrices $G_{(k)}$ for two interacting molecules shown in Eq. (15) has important implications for corrections of the one-electron DM $P_{(k)}$. Thus, products of monomolecular matrices $G_{(k)}^{(a)}$ and $G_{(k)}^{(b)}$ yield the direct sums of monomolecular intrasubset population matrices $X_{(k)+}^{(a)} \oplus X_{(k)+}^{(b)}$ and $X_{(k)-}^{(a)} \oplus X_{(k)-}^{(b)}$ within the total matrices $X_{(k)+}$ and $X_{(k)-}$. As a result, the latter take the form

$$
\begin{aligned}
& X_{(k)+}=X_{(k)+}^{(a)} \oplus X_{(k)+}^{(b)}+\delta X_{(k)+}, \\
& X_{(k)-}=X_{(k)-}^{(a)} \oplus X_{(k)-}^{(b)}+\delta X_{(k)-} .
\end{aligned}
$$

The relations of Eqs. (3), (15), and (24), in turn, allow us to conclude that any correction $P_{(k)}$ to the one-electron DM of two interacting molecules $P$ takes the form

$$
P_{(k)}=P_{(k)}^{(a)} \oplus P_{(k)}^{(b)}+\delta P_{(k)}
$$

and thereby the total DM $P$ may be represented as follows:

$$
P=P^{(a)} \oplus P^{(b)}+\delta P .
$$

Therefore, the DM of two interacting molecules consists of a sum of two matrices, the first containing the direct sum of DMs of isolated molecules and the second being a correction originating from the intermolecular interaction. This implies that intermolecular contributions to this characteristic may be studied independently from intramolecular ones.

\section{Expressions for Occupation Numbers of FOs of Two Interacting Molecules: Analysis of the Charge Redistribution}

Consider the population $x_{(+) i}$ of a certain IOFO $\varphi_{(+) i}^{a}$ of molecule $A$. In accordance with the additivity relation of Eq. (26), the expression for $x_{(+) i}$ also contains a sum of two terms, namely, the purely monomolecular term $\left(x_{(+) i}^{(a)}\right)$ and the population alteration due to interaction $\left(\delta x_{(+) i}\right)$, just the latter representing the charge redistribution under interest. To derive the expression for $\delta x_{(+) i}$, the additive form for matrices $G_{(k)}$ of Eq. (15) should be substituted into Eq. (4) and the purely monomolecular terms should be omitted.

The final form of $\delta x_{(+) i}$ contains a sum of the second-, third-, and fourth-order increments, here and below designated by superscripts (2), (3), and (4), respectively. These increments may be expressed as follows:

$$
\begin{aligned}
& \text { IVFOs(B) } \\
& \delta x_{(+) i}^{(2)}=-2 \sum_{q}^{\mathrm{IVFOS(B)}}\left(G_{(1) i q}^{(K)}\right)^{2} \\
& \delta x_{(+) i}^{(3)}=-4 \sum_{p}^{\operatorname{IVFOs}(A)} G_{(1) i p}^{(a)} \delta G_{(2) a a, i p} \\
& -4 \sum_{q}^{\operatorname{IVFOs}(B)} G_{(1) i q}^{(K)} \delta G_{(2) a b, i q} \\
& \delta x_{(+) i}^{(4)}=-4 \sum_{p}^{\operatorname{IVFOS}(A)} G_{(1) i p}^{(a)} \delta G_{(3) a a, i p}-4 \\
& \operatorname{IVFOs}(A) \\
& \times \quad \sum_{p} G_{(2) i p}^{(a)} \delta G_{(2) a a, i p}-2 \\
& \operatorname{IVFOs}(A) \\
& \times \quad \sum_{p}\left(\delta G_{(2) a, i, i p}\right)^{2}-4 \\
& \times \sum_{q}^{\mathrm{IVFOS}(B)} G_{(1) i q}^{(K)} \Delta G_{(3) a b, i q} \\
& \text { IVFOs }(B) \\
& -2 \sum_{q}\left(\delta G_{(2) a b, i q}\right)^{2},
\end{aligned}
$$

where the matrix $\Delta G_{(3) a b}$ is 

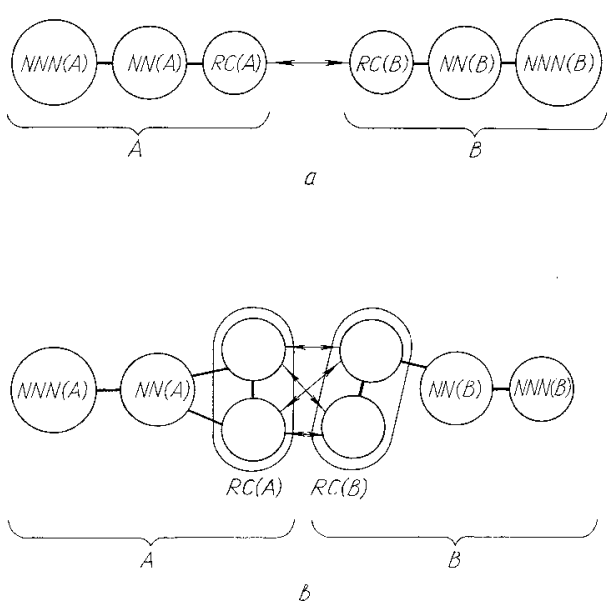

FIGURE 1. Scheme reflecting the constitution of two interacting molecules $A$ and $B$. RC, NN, and NNN stand for the reaction center of a molecule, its nearest-neighboring fragment, and its next-nearest-neighboring fragment, respectively. Intermolecular contacts are denoted by double-headed arrows. (a) Case of a local intermolecular contact when both $\mathrm{RC}(A)$ and $\mathrm{RC}(B)$ are elementary fragments. (b) Example of a nonlocal contact when both $\operatorname{RC}(A)$ and $\operatorname{RC}(B)$ consist of two elementary fragments.

$$
\begin{array}{r}
\Delta G_{(3) a b}=\delta G_{(3) a b}+G_{(1)}^{(a)} G_{(1)}^{(a)+} G_{(1)}^{(K)}+G_{(1)}^{(a)} G_{(1)}^{(L)+} G_{(1)}^{(b)} \\
+G_{(1)}^{(K)} G_{(1)}^{(b)+} G_{(1)}^{(b)}+\frac{1}{2} G_{(1)}^{(K)} G_{(1)}^{(K)+} G_{(1)}^{(K)}
\end{array}
$$

Before turning to analysis of these principal expressions, let us introduce some definitions. Let the directly contacting fragments of molecules $A$ and $B$ be called the reaction centers and be denoted by $\mathrm{RC}(A)$ and $\mathrm{RC}(B)$, respectively (the direct contact implies that the intermolecular resonance parameters $M_{i j}, K_{i q}, L_{j p}$, and $T_{p q}$ for pairs of orbitals of only these fragments take nonzero values). Further, the fragments of molecules $A$ and $B$, the orbitals of which interact directly only with those of reaction centers of their own molecules (but not with orbitals of the opposite molecule), will be referred to as the nearest-neighboring fragments and denoted by $\mathrm{NN}(A)$ and $\mathrm{NN}(B)$, respectively. Finally, the remaining parts of our systems, the orbitals of which interact directly only with those of respective NN fragments, will be called the next-nearest-neighboring fragments and acquire the notations $\mathrm{NNN}(A)$ and $\mathrm{NNN}(B)$ (Fig. 1).

It is evident that any of the above-defined fragments may contain either one or several elementary fragments defined in Section 1. In this connection, the terms for an elementary and an extended RC, $\mathrm{NN}$, and NNN fragment will be accordingly used. Further, an assumption that both $\mathrm{RC}(A)$ and $\mathrm{RC}(B)$ are elementary reaction centers implies that only two elementary fragments of molecules $A$ and $B$ actually come into contact. This case may be referred to as the case of a local intermolecular contact [Fig. 1(a)]. Alternatively, we have to do with a nonlocal contact [Fig. 1(b)]. Finally, nonzero direct intrafragmental interactions $G_{(1) i p}^{(a)}$ are peculiar only to extended fragments, in particular to extended reaction centers. Indeed, zero resonance parameters and thereby zero direct interactions correspond to orbitals of the same elementary fragment in accordance with the definition of FOs (Section 1).

\subsection{SECOND- AND THIRD-ORDER CONTRIBUTIONS TO OCCUPATION NUMBERS}

The second-order increment $\delta x_{(+) i}^{(2)}$ to the population alteration of the orbital $\varphi_{(+) i}^{a}$ defined by Eq. (27) contains only the direct intermolecular interactions $G_{(1) i q}^{(K)}$. Using Eqs. (10), (11), and (27), the respective partial transferred population between the IOFO $\varphi_{(+) i}^{a}$ and a certain IVFO $\varphi_{(-) q}^{b}$ of molecule $B$ may be defined as follows:

$$
\delta x_{(+) i,(-) q}^{(2), i n t e r}=2\left(G_{(1) i q}^{(K)}\right)^{2} .
$$

It is seen that nonzero values of $\delta x_{(+) i,(-), q}^{(2), \text { inter }}$ correspond only to pairs of orbitals described by nonzero resonance parameters $K_{i q}$ [see Eq. (18)]. Thus, a second-order intermolecular charge redistribution may be expected to take place only between orbitals of reaction centers $\mathrm{RC}(A)$ and $\mathrm{RC}(B)$. Moreover, no intramolecular charge transfer due to an intermolecular contact follows from the second-order terms. Hence, taking into account terms of power series to within the second order inclusive actually yields the local models of reactions discussed in Ref. [48] and Section 1.

Let us turn now to the third-order increment $\delta x_{(+) i}^{(3)}$ of Eq. (28). The first sum of this expression describes the population transferred from the orbital $\varphi_{(+) i}^{a}$ to IVFOs of the same molecule (intramolecular charge redistribution), whereas the second sum represents the intermolecular charge transfer between the orbital $\varphi_{(+) i}^{a}$ and IVFOs of the opposite molecule $B$. Let us start with the first of these contributions.

The third-order intramolecular partial transferred population between the IOFO $\varphi_{(+) i}^{a}$ and the IVFO $\varphi_{(-) p}^{a}$ takes the form 

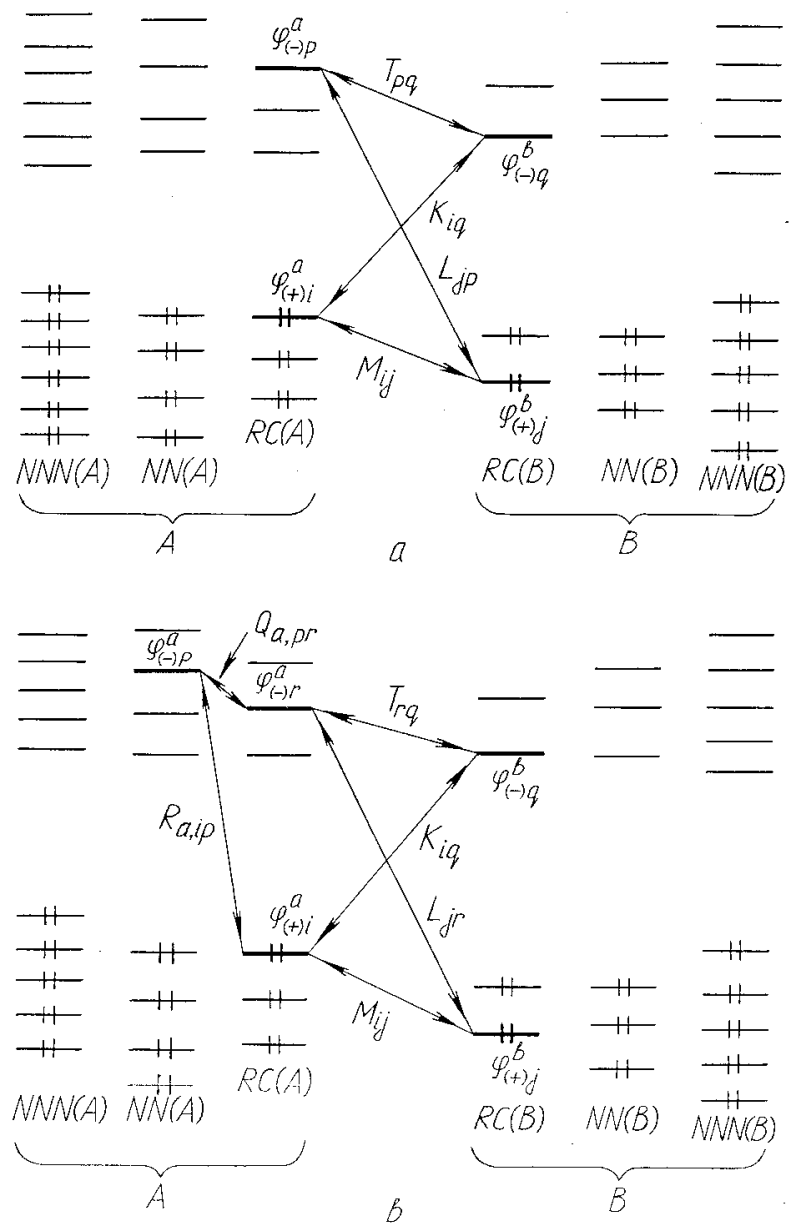

FIGURE 2. Diagram representing the energy levels of FOs corresponding to separate fragments of molecules $A$ and $B$ (RC, NN, and NNN are defined as in Fig. 1). (a) Both the electron-donating orbital $\varphi_{(+) i}^{a}$ and the electron-accepting orbitals $\varphi_{(-) p}^{a}$ and $\varphi_{(-) q}^{b}$ are localized within the reaction centers. (b) Orbital $\varphi_{(-) p}^{a}$ is localized on the nearest-neighboring fragment of molecule $A$.

$$
\delta x_{(+) i,(-) p}^{(3) \text { intra }}=4 G_{(1) i p}^{(a)} \delta G_{(2) a a, i p}
$$

and contains a product of the direct interaction of the above-mentioned orbitals $\left(G_{(1) i p}^{(a)}\right)$ and of their indirect interaction by means of an orbital of the opposite molecule $B\left(\delta G_{(2) a a, i p}\right)$ [see Eq. (23)]. Nonzero values of both factors evidently are required for a nonzero value of $\delta x_{(+) i,(-) p}^{(3) \text { intra }}$ to arise.

A considerable value of the indirect interaction $\delta G_{(2) a a, i p}$ may be expected if both the $\operatorname{IOFO} \varphi_{(+) i}^{a}$ and $\operatorname{IVFO} \varphi_{(-) p}^{a}$ belong to the reaction center of molecule $A$ [Fig. 2(a)]. To ensure a nonzero direct interaction $G_{(1) i p}^{(a)}$ between the same orbitals, however, localization of the latter on different elementary fragments of the $\mathrm{RC}(A)$ fragment is imperative. Hence, a third-order intramolecular charge redistribution may be expected to take place only inside an extended reaction center $\mathrm{RC}(A)$ provided that such a center is present in molecule $A$.

Let us turn now to the intermolecular part of the third-order population alteration $\delta x_{(+) i}^{(3)}$. The relevant partial transferred population between the IOFO $\varphi_{(+) i}^{a}$ and the IVFO $\varphi_{(-) q}^{b}$ may be expressed as follows:

$$
\delta x_{(+) i,(-) q}^{(3) \text { inter }}=4 G_{(1) i q}^{(K)} \delta G_{(2) a b, i q}
$$

where orbitals of both molecules are able to play the role of the only mediator of the indirect intermolecular interaction $\delta G_{(2) a b, i q}$. Nonzero values of both factors of the right side of Eq. (33) and thereby of the partial transferred population $\delta x_{(+) i,(-) q}^{(3), \text { inter }}$ is ensured if both $\varphi_{(+) i}^{a}$ and $\varphi_{(-) q}^{b}$ belong to the reaction centers of molecules $A$ and $B$ and at least one of these fragments is an extended $\mathrm{RC}$ in addition. Given that both $\mathrm{RC}(A)$ and $\mathrm{RC}(B)$ are elementary reaction centers, a zero value is expected for the indirect interaction $\delta G_{(2) a b, i q}$. Indeed, orbitals $\varphi_{(-) p}^{a}$ and $\varphi_{(+) j}^{b}$ [Fig. 2(a)] are not able to play the role of mediators of this interaction because of zero values of resonance parameters for pairs of orbitals $\left(\varphi_{(+) i}^{a}\right.$, $\left.\varphi_{(-) p}^{a}\right)$ and $\left(\varphi_{(+) j}^{b}, \varphi_{(-) q}^{b}\right)$ that are contained within the definition of the second-order interaction shown in Eq. (9).\} Hence, no third-order charge redistribution arises in the case of a local intermolecular contact. Otherwise, a third-order charge transfer both inside and between extended reaction centers may be expected.

On the whole, the results of this subsection indicate the primary role of the reaction center(s) in chemical reactions. Moreover, the second- and third-order local charge redistributions may be regarded as the quantum chemical analog of the supposed direct participation of the $\mathrm{RC}(A)$ and $\mathrm{RC}(B)$ fragments in certain processes.

\subsection{ANALYSIS OF SEPARATE FOURTH- ORDER INCREMENTS TO OCCUPATION NUMBERS}

Let us dwell now on the fourth-order increment $\delta x_{(+) i}^{(4)}$ shown in Eq. (29). It is seen that the first three sums of the right side of this expression describe intramolecular charge redistributions owing to the indirect influence of the opposite molecule, whereas the last two terms represent the intermolecular charge transfer. Let us consider these increments separately. 
The first increment of Eq. (29) evidently is the fourth-order analog of the first (intramolecular) term of Eq. (28). Let the respective intramolecular partial transferred population acquire an additional superscript 1, viz.

$$
\delta x_{(+) i,(-) p}^{(4) 1, \text { intra }}=4 G_{(1) i p}^{(a)} \delta G_{(3) a a, i p} .
$$

This population coincides with that defined by Eq. (32) except for the number of mediators of the indirect intramolecular interaction: The third-order interaction $\delta G_{(3) a a, i p}$ takes place by means of two mediators, at least one of them being localized on the opposite molecule $B$.

Let us assume first that the orbital $\varphi_{(+) i}^{a}$ belongs to the $\operatorname{RC}(A)$ fragment [Fig. 2(a)]. Given that it is an extended reaction center in addition, the orbital $\varphi_{(-) p}^{a}$ also may be localized on the same fragment so that $G_{(1) i p}^{(a)} \neq 0$. Then, the partial transferred population of Eq. (34) represents a fourth-order charge redistribution inside an extended reaction center due to the intermolecular contact. So far as an elementary $\operatorname{RC}(A)$ is concerned, a zero value of $\delta x_{(+) i,(-) p}^{(4) 1, \text { intra }}$ follows owing to the equality $G_{(1) i p}^{(a)}=0$.

As opposed to the third-order increment $\delta x_{(+) i,(-) p}^{(3), \text { intra }}$ of Eq. (32), however, the orbital $\varphi_{(-) p}^{a}$ may be alternatively localized on the $\mathrm{NN}(A)$ fragment of the same molecule [Fig. 2(b)] and a nonzero value of the partial transferred population $\delta x_{(+) i,(-) p}^{(4) 1 \text {,intra }}$ may be obtained. For example, a significant increment to the third-order indirect interaction $\delta G_{(3) a a, i p}$ may be ensured if the first mediator of this interaction $\left(\varphi_{(+) j}^{b}\right)$ belongs to $\operatorname{RC}(B)$, whereas the second one $\left(\varphi_{(-) r}^{a}\right)$ coincides with an IVFO of molecule $A$ localized on its reaction center $\operatorname{RC}(A)$. Again, situating the orbital $\varphi_{(-) p}^{a}$ on the $\mathrm{NNN}(A)$ fragment does not yield any contribution to the partial transferred population $\delta x_{(+) i,(-) p}^{(4) \text { intra }}$. Hence, this population may be concluded to describe an intramolecular charge transfer from the reaction center $\mathrm{RC}(A)$ toward its nearest-neighborhood $[\mathrm{NN}(A)]$ owing to the indirect influence of the approaching molecule $B$. Similarly, the orbital $\varphi_{(+) i}^{a}$ may be assumed to belong to the $\mathrm{NN}(A)$ fragment, whereas $\varphi_{(-) p}^{a}$ may be situated within $\operatorname{RC}(A)$. As a result, the partial transferred population of Eq. (34) describes an intramolecular charge transfer from the nearestneighborhood $\mathrm{NN}(A)$ toward the reaction center $\operatorname{RC}(A)$.

Thus, the partial transferred population $\delta x_{(+) i,(-) p}^{(4) 1, \text { intra }}$ may be concluded to represent an electron-donating or -accepting effect of the nearest neighborhood of molecule $A$ upon its own reaction center owing to the indirect influence of molecule $B$.

Let us turn now to the second intramolecular increment of Eq. (29). The respective partial transferred population takes the form

$$
\delta x_{(+) i,(-) p}^{(4) 2, \text { intra }}=4 G_{(2) i p}^{(a)} \delta G_{(2) a a, i p}
$$

and contains no direct interaction of the two involved orbitals $\varphi_{(+) i}^{a}$ and $\varphi_{(-) p}^{a}$ in contrast to the above-discussed first sum. Inasmuch as the only mediator of the indirect interaction $\delta G_{(2) a a, i p}$ necessarily belongs to molecule $B$, localization of both $\varphi_{(+) i}^{a}$ and $\varphi_{(-) p}^{a}$ on $\operatorname{RC}(A)$ is imperative for a nonzero value of this partial transferred population to arise. On the other hand, a considerable value of the monomolecular indirect interaction $G_{(2) i p}^{(a)}$ is possible also for two orbitals of an elementary reaction center $\operatorname{RC}(A)$ provided that an efficient mediator of this interaction may be found among orbitals of the $\mathrm{NN}(A)$ fragment. Hence, the partial transferred population of Eq. (35) describes an intramolecular charge redistribution within the reaction center $\mathrm{RC}(A)$ (including an elementary one) under influence of the opposite molecule, and orbitals of the nearest-neighboring fragment $[\mathrm{NN}(A)]$ participate in this redistribution indirectly as mediators of the indirect interaction $G_{(2) i p}^{(a)}$.

Similarly, the third sum of Eq. (29) and the relevant partial transferred population

$$
\delta x_{(+) i,(-) p}^{(4) 3, \text { intra }}=2\left(\delta G_{(2) a a, i p}\right)^{2}
$$

represents an intramolecular charge redistribution inside the reaction center of molecule $A$ mediated by orbitals of the opposite reaction center $\mathrm{RC}(B)$.

Let us consider now the last two sums of Eq. (29). Let us define the relevant partial transferred populations

$$
\delta x_{(+) i,(-) q}^{(4) 4 \text {,inter }}=4 G_{(1) i q}^{(K)} \Delta G_{(3) a b, i q}
$$

and

$$
\delta x_{(+) i,(-) q}^{(4) 5, \text { inter }}=2\left(\delta G_{(2) a b, i q}\right)^{2} .
$$

It is seen that a nonzero value of the direct interaction $G_{(1) i q}^{(K)}$ is required for the charge redistribution described by Eq. (37) to take place. This, in turn, implies that orbitals $\varphi_{(+) i}^{a}$ and $\varphi_{(-) q}^{b}$ are necessarily localized on the $\mathrm{RC}(A)$ and $\mathrm{RC}(B)$ fragments. It is also evident that orbitals of the nearest neigh- 
borhoods of these reaction centers $[\mathrm{NN}(A)$ and $\mathrm{NN}(B)$ ] may be among mediators of the third-order indirect interaction $\delta G_{(3) a b, i q}$ and thereby of its modified version $\Delta G_{(3) a b, i q}$ [see Eq. (30)]. For example, the orbitals $\varphi_{(-) p}^{a}$ and $\varphi_{(-) r}^{a}$ shown in Figure 2(b) are able to play this role. Orbitals of the $\mathrm{NNN}(A)$ and $\mathrm{NNN}(B)$ fragments, however, are not efficient mediators for the above-mentioned interaction [if we take an orbital of the $\mathrm{NN}(A)$ fragment as the first mediator of the indirect interaction $\delta G_{(3) a b, i q}$ and an orbital of the $\mathrm{NNN}(A)$ fragment as a second one, the latter does not interact with the orbital $\varphi_{(-) q}^{b}$ and thereby the final value of $\delta G_{(3) a b, i q}$ turns to zero]. Thus, Eq. (37) represents an additional charge redistribution between the two reaction centers under indirect participation of their neighboring fragments.

The last partial transferred population $\delta x_{(+) i,(-) q}^{(4) 5 \text { inter }}$ of Eq. (38) contains the square of the indirect interaction between orbitals $\varphi_{(+) i}^{a}$ and $\varphi_{(-) q}^{b}$ by means of a single mediator. A significant value of $\delta x_{(+) i,(-) q}^{(4) 5, \text { inter }}$ is ensured if either $\varphi_{(+) i}^{a}$ belongs to the $\operatorname{RC}(A)$ and $\varphi_{(-) q}^{b}$ is localized on the $\mathrm{NN}(B)$ fragment or $\varphi_{(+) i}^{a}$ is localized within the $\mathrm{NN}(A)$ fragment and $\varphi_{(-) q}^{b}$ belongs to $\mathrm{RC}(B)$. Hence, this partial population describes an indirect charge transfer between one of the two reaction centers and the nearest neighborhood of the opposite reaction center. Moreover, orbitals of the remaining reaction center situated between the electron-donating and -accepting fragments play the role of mediators for this charge redistribution.

It is seen, therefore, that an indirect participation of certain neighboring fragment in a chemical process is represented by fourth-order terms in contrast to the direct participation of the reaction center (Section 4.1). In the case of a still more remote fragment, terms of even higher orders evidently are required to describe the relevant effects. Thus, extinction of an indirect influence may be expected when the distance between the given fragment and the respective reaction center grows.

The last prediction based on the above results is as follows: At the early stage of the reaction when the intermolecular resonance parameters are relatively small, the lower-order terms and thereby the structures of the reaction centers are likely to play the decisive role. When the process goes on, however, the higher-order terms and thereby the influence of the neighboring fragments become relatively more important.

\section{Applicability of the Approach Suggested to Specific Chemical Problems}

Comparison of partial transferred populations of Eqs. (31)-(38) referring to alternative routes of certain reaction may serve to demonstrate the aboveexpected applicability. To this end, a specific model of the given process is required, including particular structures both of the participating fragments (RC, NN, and NNN) and of FOs along with respective energy levels. Moreover, confinement to certain number of principal FOs and their interactions (resonance parameters) also is advisable, especially when looking for qualitative conclusions. For example, a single initially occupied (vacant) orbital $\varphi_{(+) N}\left(\varphi_{(-) E}\right)$ may be successfully used to represent a nucleophilic (electrophilic) reagent $B$ [44, 49] when comparing different directions of its attack upon the same reactant $A$ [such a one-orbital model implies that the reagent $B$ consists only of an elementary reaction center $\mathrm{RC}(B)]$.

For illustration, let us dwell first on the extended model of the $S_{N} 2$ reaction between a substituted alkane $\left(\mathrm{Z}-\mathrm{C}_{\alpha} \mathrm{H}_{2}-\mathrm{C}_{\beta} \mathrm{H}_{2}-\ldots\right)$ and nucleophile $(\mathrm{Nu})$ suggested recently [44], where $Z$ stands for a heteroatom (nucleofuge). As opposed to the abovediscussed local model [1, 6, 29], intermolecular resonance parameters between the orbital of the reagent $\left(\varphi_{(+) N}\right)$ and orbitals of the $C_{\alpha}-C_{\beta}$ and/or $\mathrm{C}_{\alpha}-\mathrm{H}$ bonds of the reactant also are taken into account here. This evidently implies an assumption about an extended reaction center $\mathrm{RC}(A)$ containing four bonds (viz. the $\mathrm{C}_{\alpha}-\mathrm{Z}$ bond and its three geminal neighbors) and thereby about a nonlocal intermolecular contact. Application of this new model allowed us to distinguish between the efficiencies of the frontal and back attacks of nucleophile even if the respective direct intermolecular interactions between the orbital $\varphi_{(+) N}$ and the antibonding orbital of the $\mathrm{C}_{\alpha}-\mathrm{Z}$ bond $\left(\varphi_{(-) 1}\right)$ take coinciding absolute values. The point is that the indirect interactions between orbitals $\varphi_{(+) N}$ and $\varphi_{(-) 1}$ by means of orbitals of the $\mathrm{C}_{\alpha}-\mathrm{C}_{\beta}\left(\mathrm{C}_{\alpha}-\mathrm{H}\right)$ bonds playing the role of mediators contribute significantly to a greater efficiency of the back attack of nucleophile as compared to the frontal one (it is the back attack that is commonly assumed to give rise to a subsequent reaction $[1-6,29,30])$.

The immediate reasons for such a result are as follows: The above-mentioned assumption of coincidence between absolute values of direct interac- 
tions $\left(G_{(1) i q}^{(K)}\right)$ for the frontal and back attacks of nucleophile evidently implies an equality between respective second-order partial transferred populations defined by Eq. (31) and thereby makes a comparison of indirect increments to relative efficiencies of both attacks easier (that is why the assumption of uniform direct interactions was invoked). Further, the correction $\delta x_{(+) N,(-) 1}^{(3) \text { inter }}$ of Eq. (33) describing the indirect intermolecular charge transfer between orbitals $\varphi_{(+) N}$ and $\varphi_{(-) 1}$ by means of orbitals of the $\mathrm{C}_{\alpha}-\mathrm{C}_{\beta}\left(\mathrm{C}_{\alpha}-\mathrm{H}\right)$ bonds proved to be of the largest absolute value among the third-order partial transferred populations of Eqs. (32) and (33). The most important aspect, however, consists of opposite signs of the above-mentioned principal correction for alternative directions of the attack, namely, a positive (negative) sign of correction $\delta x_{(+) N,(-) 1}^{(3) \text {,inter }}$ corresponds to the back (frontal) attack of nucleophile (different signs of direct interactions $G_{(1) N 1}^{(K)}$ along with coinciding signs of indirect interactions $\delta G_{(2) a b, N 1}$ give rise to this result). Consequently, the third-order increment $\delta x_{(+) N,(-) 1}^{(3), \text { inter }}$ is added (subtracted) to (from) the positive secondorder partial transferred population $\delta x_{(+) N,(-) 1}^{(2), \text { inter }}$ and thereby a larger (smaller) value of the total population acquired by the $\mathrm{Z}-\mathrm{C}_{\alpha}$ bond is obtained for the back (frontal) attack of nucleophile. It is natural to assume that the more population the nucleofuge acquires, the easier it leaves. Hence, a greater efficiency of the back attack due to indirect increments has been concluded.

Let us turn now to the electrophilic addition $\left(\mathrm{Ad}_{E} 2\right)$ reaction of substituted ethenes $\left(\mathrm{H}_{2} \mathrm{C}_{\beta}=\mathrm{C}_{\alpha}\right.$ $\mathrm{HX}$ ). In the simplest qualitative model of the process [49], the electron-donating or -accepting substituent $X(X=D, A)$ may be represented by a single IOFO $\left(\varphi_{(+) d}\right)$ or a single IVFO $\left(\varphi_{(-) a}\right)$. The ethene fragment may be accordingly described by the bonding and antibonding $\pi$-orbitals of the $\mathrm{C}_{\alpha}=\mathrm{C}_{\beta}$ bond, further referred to as $\varphi_{(+) e}$ and $\varphi_{(-) e}$. The electrophilic reagent $\left(E^{+}\right)$may be represented by a single IVFO $\varphi_{(-) E}$ as previously. The initial attacks of this reagent upon the $C_{\beta}$ and $C_{\alpha}$ atoms present the two routes of the process under comparison, and these will be called a terminal and an internal attack, respectively. Coincidence of the second-order corrections of Eq. (31) for these alternative directions of the attack may be ensured by choice of uniform values for intermolecular resonance parameters between the orbital $\varphi_{(-) E}$ and the $2 \mathrm{p}_{z} \mathrm{AO}$ of the carbon atom under attack. Similarly to the above-discussed $S_{N} 2$ reaction, such an assumption allows us to reveal the dependence of charge redis- tribution among the three fragments of the system (viz. the $\mathrm{C}_{\alpha}=\mathrm{C}_{\beta}$ bond, the substituent $\mathrm{X}$, and the reagent $\mathrm{E}^{+}$) only upon their mutual spatial arrangement. As opposed to the $S_{N} 2$ reaction, however, a local intermolecular contact may be assumed to be peculiar to the $\operatorname{Ad}_{E} 2$ process (resonance parameters between the orbital $\varphi_{(-) E}$ and that of the substituent $\mathrm{X}$ take small values and may be ignored). Thus, our reactant $A$ (the substituted ethene) contains an elementary reaction center $\mathrm{RC}(A)$ consisting of the $\mathrm{C}_{\alpha}=\mathrm{C}_{\beta}$ bond only. Consequently, the third-order partial transferred populations of Eqs. (32) and (33) vanish and thereby the $\operatorname{Ad}_{E} 2$ reaction proves to be governed by fourth-order corrections defined by Eqs. (34)-(38).

For the case of an electron-donating substituent $(X=D)$, the following principal fourth-order corrections have been revealed: (1) the partial transferred population $\delta x_{(+) d,(-) e}^{(4) 1 \text { intra }}$ defined by Eq. (34) and describing the alteration in the electron-donating effect of the substituent D upon the $\mathrm{C}_{\alpha}=\mathrm{C}_{\beta}$ bond under an indirect participation of electrophile (the orbital $\varphi_{(-) E}$ is among the mediators of the indirect interaction $\left.\delta G_{(3) a a, d e}\right) ;(2)$ the increment $\delta x_{(+) e,(-) E}^{(4) 4 \text {,inter }}$ following from Eq. (37) and representing the alteration in the electron-donating effect of the $\mathrm{C}_{\alpha}=\mathrm{C}_{\beta}$ bond toward electrophile under an indirect participation of the orbital $\varphi_{(+) d}$ of the substituent D [the latter is among the mediators of the third-order interaction $\Delta G_{(3) a b, e E}$ of Eq. (30)]; and (3) the partial transferred population $\delta x_{(+) d,(-) E}^{(4), \text { inter }}$ defined by Eq. (38) and describing the indirect charge transfer between the orbital of the substituent $\left(\varphi_{(+) d}\right)$ and that of electrophile $\left(\varphi_{(-) E}\right)$.

Comparative studies of these increments for the internal and terminal position of electrophile with respect to the substituent $\mathrm{D}$ showed that positive (negative) corrections $\delta x_{(+) d,(-) e}^{(4) 1, \text { intra }}$ and $\delta x_{(+) e,(-) E}^{(4) 4, \text { inter }}$ and a large (small) positive value of the remaining correction $\delta x_{(+) d,(-) E}^{(4) 5, \text { inter }}$ are peculiar to the terminal (internal) position of the reagent. Thus, it is the terminal attack of electrophile upon the molecule $\mathrm{H}_{2} \mathrm{C}_{\beta}=\mathrm{C}_{\alpha} \mathrm{HD}$ that is accompanied by more extended transferred population between any pair of fragments of the whole reacting system and thereby a larger stabilization energy [35]. This result is inline with the observed higher reactivity of the terminal position of the molecule $\mathrm{H}_{2} \mathrm{C}_{\beta}=\mathrm{C}_{\alpha} \mathrm{HD}$ with respect to electrophile (cf. the so-called Markovnikov rule $[1-4,6])$.

For an electrophilic attack upon an $A$-substituted ethene $\left(\mathrm{H}_{2} \mathrm{C}_{\beta}=\mathrm{C}_{\alpha} \mathrm{HA}\right)$, two partial transferred populations determine the difference in reactivities of 
carbon atoms, namely, $\delta x_{(+) e,(-) E}^{(4) 4, \text { inter }}$ and $\delta x_{(+) e,(-) a}^{(4) 1 \text {,intra }}$. The first of these increments describes the alteration in the electron-accepting effect of electrophile upon the $\mathrm{C}_{\alpha}=\mathrm{C}_{\beta}$ bond owing to the presence of the electron-accepting substituent $A$, whereas the second one represents an analogous alteration in the electron-accepting effect of the orbital $\varphi_{(-) a}$. Moreover, an internal (terminal) attack of electrophile was shown to be characterized by positive (negative) values of these principal corrections and thereby by more (less) extended electron-accepting effects of both acceptors. This result indicates an internal addition of electrophile and also is inline with experimental facts $[2-4,6]$.

Hence, different relative reactivities of carbon atoms in substituted ethenes proved to be related to dissimilar indirect influences of the substituent for alternative directions of an electrophilic attack. Moreover, the larger reactivities of the terminal (internal) carbon atoms vs. the remaining ones for the cases $\mathrm{X}=\mathrm{D}(\mathrm{X}=\mathrm{A})$ are accompanied by more (less) efficient indirect influencies of the substituent $\mathrm{D}(A)$.

On the whole, the above-discussed two examples demonstrate applicability of the approach suggested to specific chemical problems and its possibilities to predict relative reactivities for alternative routes of reactions.

\section{Conclusions}

The electron density redistributions among separate fragments of interacting molecules studied in this article are expressed algebraically in terms of fragmental orbitals and their direct and indirect interactions. At the same time, our final results are in-line with the principal concepts of the classic chemistry concerning reactivity. Thus, the following classic intuition-based hypotheses acquire an additional quantum chemical support:

1. The assumption about different roles of the reaction center and of its nearest neighborhood in a chemical process.

2. The expectation about extinction of the indirect influence of the given fragment upon the reactivity of the whole compound when the distance between this fragment and the respective reaction center grows.

3. The assumption about a variable relative im- portance of the neighboring fragments vs. that of the reaction center during the process.

Further, the above-obtained results yield quantum chemical representations of the direct and indirect participation of separate fragments in a chemical process. Thus, local redistributions of electron density among fragmental orbitals inside and between the supposed reaction centers describe the consequences of their direct participation in the given reaction. Alternatively, an indirect influence of certain nearest-neighboring (NN) fragment (e.g., of a substituent) upon the reactivity of the whole compound is shown to consist of three principal quantum chemical components:

1. The NN fragment exerts an additional electron-donating or -accepting effect upon the reaction center of its own molecule under influence of the approaching reaction center of the opposite molecule because the latter offers its orbitals as mediators for certain indirect intramolecular interaction.

2. The NN exerts an analogous effect upon the reaction center of the opposite molecule owing to an ability of orbitals of its own reaction center to mediate some indirect intermolecular interactions.

3. The NN fragment offers its orbitals as mediators for indirect interorbital interactions that give rise to additional electron density redistributions both inside the reaction center of its own molecule and between the two reaction centers.

It is also noteworthy that both the local charge redistributions representing consequences of direct participation of the reaction centers in the given process and the above-enumerated three principal components of the indirect influence of a substituent depend on the spatial arrangement of the reagent with respect to reactant. Thus, relative efficiencies of alternative routes of the process (if any) may be predicted on the basis of comparison of extents of these effects (see, e.g., [44, 49]).

The scope of validity of the approach suggested evidently is determined by the range of convergence of the power series for the DM. For particular types of molecules, the rates of convergence of this series have been studied in Refs. [36-38, 40, 41, 43]. It turned out that a rapid convergence is primarily ensured for molecules consisting of weakly inter- 
acting (quasitransferable) elementary fragments, e.g., substituted alkanes [37, 38], aliphatic conjugated hydrocarbons of small and medium size along with their derivatives [40], as well as substituted benzenes [41, 43]. Hence, reactions of just these compounds are likely to be studied most successfully using the semilocalized approach. The results concerning an $S_{N} 2$ reaction of substituted alkanes [44] and an $\operatorname{Ad}_{E} 2$ reaction of substituted ethenes [49] overviewed in Section 5 support this expectation. Finally, the above-discussed parallelism between our results and the classic concepts promotes an expectation that the same scope of validity refers to these concepts as well.

The direct way of obtaining the one-electron density matrix on the basis of solution of the commutation equation [36-43] used in this article is closely related [36] to the matrix form of the Brillouin theorem, underlying the direct method of derivation of noncanonical (localized) MOs (LMOs) without invoking CMOs [36, 39, 47, 50-54]. This relation, in turn, allows the relevant two problems to be considered as parts of the same noncanonical method of MOs (NCMO method) [39, 40, 47]. Moreover, the one-electron DM and the respective LMO representation matrix have been expressed in terms of the same submatrices (blocks) and thereby proved to be alternative forms of the localized description of electronic structures of molecules [36]. In this context, the above-obtained results may be regarded as an extension of the NCMO method and thereby of the localized description to investigation of chemical reactivity.

The latter conclusion allows us to reformulate the scope of validity of the approach suggested and thereby of the above-discussed classic chemical concepts in terms of LMOs. Indeed, the power series for the $\mathrm{DM}$ and that for the LMO representation matrix are expected to converge (or diverge) simultaneously [40]. Convergence of these series, in turn, implies existence of LMOs of the basis-orbital-andtail structure for the given compound(s), i.e., of LMOs characterized by a large extent of localization. Inasmuch as LMOs are alternatively obtainable by transforming the set of CMOs using various localization criteria [54], localizability of CMOs of certain molecule(s) also is a criterion of applicability of the concept of the reaction center and its neighborhood when discussing the relevant reactions.

The NCMO and CMO methods have been considered as complementary approaches for investigation of electronic structures of molecules [40]. The same conclusion is likely to refer to the relevant studies of chemical reactivity as well: In the standard perturbative approaches based on CMOs [1, 29-32], emphasis is laid on search of that characteristic of the reactant molecule as a whole that determines its subsequent reactivity (cf. the socalled reactivity indices [31, 32]). When turning to the above-suggested approach, emphasis is replaced on studies of roles of separate fragments of both participants of the reaction in the given process. General results concerning the role of the reaction center and its neighborhood are obtained is this case.

\section{References}

1. Klopman, G., ed. Chemical Reactivity and Reaction Paths; John Wiley \& Sons: New York, 1974.

2. March, J. Advanced Organic Chemistry, Reactions, Mechanisms and Structure; Wiley Interscience: New York, 1985.

3. Carroll, F. A. Perspectives on Structure and Mechanism in Organic Chemistry; Brooks/Cole: Pacific Grove, CA, 1998.

4. Becker, H. G. O. Einfürung in die Elektronentheorie Organisch Chemischen Reaktionen; Deutscher Verlag der Wissenschaften: Berlin, 1974.

5. Edenborough, M. Organic Reaction Mechanisms. A Step by Step Approach; Taylor and Francies: London, 1999.

6. Dnieprovskii, A. S.; Temnikova, T. I. Theoretical Fundamentals of Organic Chemistry; Khimia: Leningrad, 1991 (in Russian).

7. Vasiljeva, N. V. Theoretical Introduction into Organic Synthesis; Prosveshtchenie: Moscow, 1976 (in Russian).

8. Pauling, L.; Wheland, G. W. J Chem Phys 1933, 1, 362.

9. Ri, T.; Eyring, H. J Chem Phys 1940, 8, 433.

10. Pullman, A.; Pullman, B. Experientia 1946, 2, 364

11. Dewar, M. J. S. Trans Faraday Soc 1946, 42, 764.

12. Coulson, C. A. Discuss Faraday Soc 1947, 2, 9.

13. Coulson, C. A.; Longuet-Higgins, H. C. Proc Roy Soc Lond 1947, A191, 39; Coulson, C. A.; Longuet-Higgins, H. C. Proc Roy Soc Lond 1947, A192, 16.

14. Wheland, G. W. J Am Chem Soc 1942, 64, 900.

15. Dewar, M. J. S. J Am Chem Soc 1952, 74, 3357.

16. Fukui, K.; Yonezawa, T.; Shingu, H. J Chem Phys 1952, 20, 722.

17. Mulliken, R. S. J Am Chem Soc 1952, 74, 811.

18. Brown, R. D. J Chem Soc 1959, 2232.

19. Fukui, K.; Fujimoto, H. Bull Chem Soc Jpn 1968, 41, 1989; Fukui, K.; Fujimoto, H. Bull Chem Soc Jpn 1969, 42, 3399.

20. Fujimoto, H.; Yamabe, S.; Fukui, K. Bull Chem Soc Jpn 1971, 44, 2936.

21. Fukui, K. Acc Chem Res 1971, 4, 57.

22. Fukui, K. Angew Chem Int Ed 1982, 21, 801.

23. Klopman, G.; Hudson, R. F. Theor Chim Acta 1967, 8, 165.

24. Klopman, G. J Am Chem Soc 1968, 90, 223.

25. Salem, L. J Am Chem Soc 1968, 90, 543, 553. 


\section{GINEITYTE}

26. Devaquet, A.; Salem, L. J Am Chem Soc 1969, 91, 3793.

27. Devaquet, A. Mol Phys 1970, 18, 233.

28. Sustman, R.; Binch, G. Mol Phys 1971, 20, 1, 9.

29. Dewar, M. J. S.; Dougherty, R. C. The PMO Theory of Organic Chemistry; Plenum Press: New York, 1975.

30. Tedder, J. M.; Nechvatal, A. Pictorial Orbital Theory; Pitman: London, 1985.

31. Zhidomirov, G. M.; Bagaturiants, A. A.; Abronin, I. A. Applied Quantum Chemistry. Calculations of Chemical Reactivity and Mechanisms of Reactions; Khimia: Moscow, 1979 (in Russian).

32. Basilevskii, M. V. The MO Method and Reactivity of Organic Compounds; Khimia: Moscow, 1969 (in Russian).

33. Fleming, I. Frontier Orbitals and Organic Chemical Reactions; Wiley: Chichester, UK, 1976.

34. Traven, V. F. Electronic Structure and Properties of Organic Compounds; Khimia: Moscow, 1989 (in Russian).

35. Gineityte, V. J Mol Struct Theochem 2002, 585, 15.

36. Gineityte, V. J Mol Struct Theochem 1995, 343, 183.

37. Gineityte, V. J Mol Struct Theochem 1998, 430, 97.

38. Gineityte, V. J Mol Struct Theochem 1998, 434, 43.
39. Gineityte, V. Int J Quantum Chem 1998, 68, 119.

40. Gineityte, V. J Mol Struct Theochem 1999, 487, 231.

41. Gineityte, V. J Mol Struct Theochem 2000, 507, 253.

42. Gineityte, V. Int J Quantum Chem 2000, 77, 534.

43. Gineityte, V. J Mol Struct Theochem 2001, 546, 107.

44. Gineityte, V. J Mol Struct Theochem 2001, 541, 1.

45. Gineityte, V. J Mol Struct Theochem 2000, 532, 257.

46. Zahradnik, R.; Polak, R. Elements of Quantum Chemistry; Plenum Press: New York, 1980.

47. Gineityte, V. Int J Quantum Chem 1999, 72, 559.

48. Gineityte, V. J Mol Struct Theochem 1999, 465, 183.

49. Gineityte, V. J Mol Struct Theochem 2002, 588, 99.

50. Daudey, J. P. Chem Phys Lett 1974, 24, 574.

51. Mayer, I. Chem Phys Lett 1982, 89, 390.

52. Surján, P. R.; Mayer, I.; Kertész, M. J Chem Phys 1982, 77, 2454

53. Mayer, I.; Surján, P. R. J Chem Phys 1984, 80, 5649.

54. Chalvet, O., ed. Localization and Delocalization in Quantum Chemistry, Atoms and Molecules in the Ground State, vol. 1; Reidel: Dordrecht, The Netherlands, 1975. 